Fibroepithelial breast lesion: when sequencing can help to make a clinical decision.

\title{
A Case Report.
}

Giacomo Montagna ${ }^{1}$, Charlotte K. Y. Ng${ }^{2,3}$, Tatjana Vlajnic ${ }^{3}$, Viola Paradiso ${ }^{3}$, Sophie Dellas ${ }^{4}$, Hubertina Reina ${ }^{1}$, André Kind ${ }^{1}$, Walter Paul Weber ${ }^{1}$, Salvatore Piscuoglio ${ }^{3}$ and Christian Kurzeder $^{1}$

${ }^{1}$ Breast Center, University Hospital Basel, Basel, Switzerland

${ }^{2}$ Department of Biomedicine, University of Basel, Basel, Switzerland.

${ }^{3}$ Institute of Pathology, University Hospital Basel, Basel, Switzerland

${ }^{4}$ Department of Radiology, University Hospital Basel, Basel, Switzerland

Keywords: fibroepithelial lesion, phyllodes tumor, MED12, TERT promoter

Corresponding Author and person to whom reprint requests should be addressed:

Giacomo Montagna, MD

University Hospital Basel

Breast Center

4031, Spitalstrasse 21

Switzerland

Phone: +4161328 7991

Fax: +41612659185

E-mail: giacomo.montagna@usb.ch

ORCID ID: 0000-0003-3511-369X 


\section{Abstract:}

Mammary fibroepithelial lesions are biphasic neoplasms that comprise a wide spectrum of tumors ranging from indolent fibroadenomas to rare malignant phyllodes tumors. The histological distinction between the two on core needle biopsy can be challenging. Recently, TERT promoter mutations and gene amplifications have been used to differentiate fibroadenomas from phyllodes tumors. We present the case of a 59-year old woman initially diagnosed with a fibroepithelial lesion who refused surgery and presented 13 months later with a disfiguring borderline phyllodes tumor. Sequencing of the early fibroepithelial lesion and of the phyllodes tumor showed MED12 hotspot (c.131G>A) and TERT promoter (c.-124C>T) somatic mutations, suggesting that the lesion was already a phyllodes tumor at time of the initial biopsy. The characterization of somatic mutations on core needle biopsy can help to better characterize fibroepithelial lesions and therefore increase diagnostic accuracy. 


\section{Clinical Practice points:}

- Breast fibroepithelial lesions comprise a wide spectrum of tumors ranging from indolent fibroadenomas to malignant phyllodes tumors. The histological distinction between the two on core needle biopsy can be challenging.

- There is a need for molecular markers to better discriminate subclasses of breast fibroepithelial lesions on core needle biopsy to tailor clinical management.

- Recently, TERT promoter mutations and gene amplifications have been used to differentiate fibroadenomas from phyllodes tumors.

- This report describes a 59-year old woman diagnosed with a fibroepithelial lesion of the left breast who refused surgery and presented 13 months later with a disfiguring borderline phyllodes tumor that required mastectomy. Sequencing of the early FEL and of the PT showed MED12 hotspot (c.131G>A) and TERT promoter (c.-124C>T) somatic mutations, suggesting that the lesion at time of the initial biopsy was already a phyllodes tumor.

- The characterization of somatic mutations on core needle biopsy can help to better characterize fibroepithelial lesions and therefore increase diagnostic accuracy. 


\section{Introduction:}

Breast fibroepithelial lesions (FELs) are composed of biphasic proliferation of both epithelial and stromal elements and comprise a wide spectrum of tumors ranging from indolent fibroadenomas (FA) to malignant phyllodes tumors (PT). ${ }^{1}$ Whilst FAs are common benign tumors and are usually managed conservatively, PTs are rare (accounting for $\sim 2.5 \%$ of all mammary FELs and for $1 \%$ of all breast cancers) and may recur locally or even metastasize to distant sites. ${ }^{1}$

The histologic distinction between FAs and PTs on core needle biopsy (CNB) can be challenging. ${ }^{2}$ Pathologists may designate a FEL and add a comment of concern such as "cannot rule out phyllodes" or "increased stromal cellularity" if features of phyllodes are present but not definitive. ${ }^{3}$ However, the exact characterization of a FEL is clinically important as the management may range from observation to wide surgical resection ( $\geq 1 \mathrm{~cm}$ margin). ${ }^{2,4}$ As it may be impossible to distinguish FA from benign or borderline PT on CNB due to overlapping histological features, ${ }^{2,3,5}$ excision is recommended in many patients resulting in potential overtreatment. $^{2,5,6}$ A selective surgical approach based on clinical, radiological and pathological features is therefore recommended. ${ }^{4,7}$

FAs and PTs share not only histologic similarities, but also genetic features. ${ }^{8}$ Recurrent somatic mutations affecting exon 2 of MED12 have been identified in both lesions. ${ }^{9-12}$ Additionally, recent genomic analyses of FAs and PTs ${ }^{12}$ demonstrated that PTs are genetically more advanced and display a higher mutational burden than FAs. ${ }^{9}$ Furthermore, there have been reports of progression from FAs to PTs, ${ }^{8,13,14}$ with MED12 mutation as the founder genetic event and the subsequent acquisition of additional somatic genetic alterations such as TERT promoter mutations in the PTs. ${ }^{8}$ 
Here, we describe the case of a 59-year old woman initially diagnosed with a FEL with a TERT promoter mutation who refused surgery and presented 13 months later with a disfiguring borderline PT.

\section{Case report:}

In May 2016, a 59-year old woman with no personal history of breast or ovarian cancer was diagnosed with polymyositis. Clinical examination revealed an asymmetry of the left breast and a $\sim 6 \mathrm{~cm}$ palpable mass on the upper external quadrant (Figure 1A) but no lymphadenopathies. Mammogram (Figure 1B) and ultrasound confirmed a $5.5 \times 5 \mathrm{~cm}$ mass in the upper external quadrant of the left breast and a $0.6 \times 0.7 \mathrm{~cm}$ mass in the upper external quadrant of the right breast. CNB of both lesions was performed for diagnostic purposes. Histologic examination revealed a FA on the right side (classified as B2-lesion) (Figure 1C) and a FEL with increased stromal cellularity on the left side (classified as B3-lesion) (Figure 1D). Surgical excision of the FEL lesion was scheduled, but shortly before surgery the patient canceled the operation and was lost to follow up.

Thirteen months later (June 2017), the patient presented to the emergency department with disfiguring swelling of the left breast (Figure 2A). On physical examination, the left breast was massively enlarged and tender with a flattened nipple, engorged superficial vessels, and deeply erythematous skin (Figure 2A). Breast ultrasound and MRI (Figure 2B) showed a 16 $\mathrm{cm}$ heterogeneous mass filling the entire left breast with an extensive central fluid collection without suspected lymphadenopathies (Figure 2B). A diagnostic puncture of $1200 \mathrm{ml}$ of bloody fluid was sent for cytology and microbiological tests that revealed no bacterial growth and no cellular atypia. CNB showed a myofibroblastic proliferation without an epithelial component. Differential diagnosis included a PT (stromal component only) or a non-specific reactive inflammatory process. After a multidisciplinary discussion, due to the large size of the tumor, mastectomy was performed. Based on the presence of focal stromal overgrowth, increased mitoses (7 per $10 \mathrm{HPF}$ ) and focally invasive tumor borders (Figure 2C-D), the diagnosis of a borderline PT was made on final histology. The patient recovered well, without complications. 
Thirteen months after treatment, the patient continued to do well with no evidence of disease recurrence.

To correlate the genomic profile of the tumor with its clinical evolution, massively parallel sequencing of 32 genes commonly altered in breast cancer (Supplementary Table 1) was performed, and analyzed as previously described, ${ }^{15}$ on tissues from the FA (right breast), the FEL and the PT (left breast). No somatic mutations were identified in any of the genes recurrently mutated in breast cancer. Subsequently, all three lesions were subjected to Sanger sequencing for the MED12 hotspot and for TERT promoter somatic mutations. Again, no mutation was identified in the FA in the right breast (Figure $3 \mathrm{~A}$ ). However, we identified a MED12 hotspot (c.131G>A) and TERT promoter (c. $-124 \mathrm{C}>\mathrm{T}$ ) mutations in both the early B3 lesion and in the subsequent lesion after 13 months, suggesting the progression of the early lesion. This observation was further supported by their shared copy number alterations on chromosomes 11, 12 and 21, and the subsequent acquisition of copy number loss of $A K T 1$ and TP53 in the later lesion (Figure 3B).

\section{Discussion:}

This 59-year old woman was diagnosed with a FEL with increased stromal cellularity (B3lesion). The uncertain malignant potential was discussed with the patient in detail and surgical excision was scheduled. Given the absence of "cancer cells" on the CNB, the patient perceived the lesion as not dangerous. The patient canceled the scheduled operation and follow up was lost. The disfiguring evolution of the breast lesion and the inconclusive diagnosis on the second CNB 13 months later led to a mastectomy without reconstruction.

Currently, there are no absolute criteria to recommend the excision of FELs when definitive diagnosis of a PT is not rendered after CNB. ${ }^{2,7}$ Many authors advocate reliance on pathologists' comments and concordance assessment as important considerations to guide clinical management of FELs. ${ }^{3,16}$ Van Osdol et al. recently reported a sensitivity and specificity of $82 \%$ and $93 \%$ respectively for a pathologist comment of concern. ${ }^{7}$ Nevertheless, only $18-38 \%$ of 
patients who received excision for a FEL had a PT on definitive histology. $3,17,18$ The sometimes extreme difficulty to distinguish PTs from cellular FA, particularly in a limited tissue sample of a CNB, has also been acknowledged in the latest WHO classification, ${ }^{1}$ which states that in inconclusive cases, the diagnosis of a cellular fibroepithelial neoplasm may be appropriate, recognizing the inability to classify these lesions correctly ${ }^{19}$.

Recently, it has been suggested that TERT alterations may assist in the differential diagnosis between FAs and PTs, as TERT promoter mutations and gene amplifications are frequent in PTs but are absent in FAs, and their frequency increases according to the grade of the PT (18\% in benign, $57 \%$ in borderline and $64 \%$ in malignant PTs). ${ }^{8,20}$ Piscuoglio et al. concluded that sequencing and gene copy number analysis differentiate FAs from PTs with a $100 \%$ specificity and $100 \%$ positive predictive value. ${ }^{8}$ The finding of a TERT promoter mutation in the early B3 FEL on the left side would suggest it was also a PT.

Genomic profiling has also furthered our understanding of the etiopathology of PTs. Pareja et al. ${ }^{21}$ have recently studied PTs with and without FA-like areas and hypothesized that borderline and malignant PTs might follow two distinct evolutionary pathways, according to MED12 status. In the MED12-mutant pathway, MED12 exon 2 mutations are posited to lead to the development of a benign FEL, which, upon the occurrence of additional genetic alterations affecting TERT and/or other cancer gene, may progress to a borderline or malignant PT. In the MED12-independent pathway, borderline or malignant PTs might arise de novo, through acquisition of genetic alterations targeting cancer genes such as TERT and/or EGRF and other tumor suppressor genes. ${ }^{21}$ The presence of both the MED12 and TERT mutations in our current case would suggest it likely occurred through the MED12-mutant pathway, followed by the subsequent acquisition of the TERT promoter mutation, as well as additional copy number alterations. 
The evolution from FA to PTs rarely occurs and has been reported in the literature as a slow process $^{22,23}$. On the other hand, PTs are often reported as rapidly growing ${ }^{24}$. Whether growth rate differs according to the expression of MED12 is unknown. In the present case, the patient presented with a $5 \mathrm{~cm}$ FEL, with MED12 and TERT promoter mutations, which rapidly evolved into a disfiguring borderline PT, which needed mastectomy.

\section{Conclusion:}

Recent studies have suggested that TERT promoter hotspot mutation and/or amplification may drive PT progression from a benign lesion such as FA, which represents an example of how genomic profiling may inform the clinical management of FELs. The current case demonstrates the natural course of a borderline PT after refusal of surgery. Knowing that the lesion had a TERT mutation and therefore a high likelihood of being a PT might have had encouraged the patient to undergo surgical excision and thus would have spared her the burden of a mastectomy. Further studies are needed to evaluate the impact of massively parallel sequencing on the outcome of patients with FELs.

FINANCIAL SUPPORT: S.P. is supported by Swiss National Science Foundation (Ambizione grant number PZ00P3_168165). V.P. is supported by the Swiss Centre for Applied Human Toxicology (SCAHT). Funding bodies had no role in the design of the study, collection, analysis and interpretation of the data or the writing of the manuscript.

ACKNOWLEDGMENTS: None

CONFLICT OF INTEREST: The authors declare no potential conflicts of interest.

AUTHOR'S CONTRIBUTION: G.M., C.K.Y.N., S.P. and C.K. conceived and supervised the study; V.P. performed DNA extraction, library preparation, sequencing and Sanger Sequencing; T.V. performed histologic review; S.D reviewed radiological images, G.M., A.K, 
H.R., W.P.W. and C.K. provided samples and clinical data. G.M., C.K.Y.N. and S.P. analyzed the results and wrote the manuscript.

\section{References:}

1. Lakhani SR, Ellis IO, Schnitt SJ, Tan PH, van de Vijver MJ. WHO Classification of Tumours of the Breast. Vol 4. Lyon: IARC Press; 2012.

2. Karim RZ, O'Toole SA, Scolyer RA, et al. Recent insights into the molecular pathogenesis of mammary phyllodes tumours. J Clin Pathol. 2013;66(6):496-505.

3. Resetkova E, Khazai L, Albarracin CT, Arribas E. Clinical and radiologic data and core needle biopsy findings should dictate management of cellular fibroepithelial tumors of the breast. Breast J. 2010;16(6):573-580.

4. NCNN guidelines for Phylloides tumors. http://www.nenn.org National Comprehensive Cancer Network website. Version 2.2017. Accessed May, 19, 2018.

5. Tan PH, Ellis IO. Myoepithelial and epithelial-myoepithelial, mesenchymal and fibroepithelial breast lesions: updates from the WHO Classification of Tumours of the Breast 2012. J Clin Pathol. 2013;66(6):465-470.

6. Jacobs TW, Connolly JL, Schnitt SJ. Nonmalignant lesions in breast core needle biopsies: to excise or not to excise? Am J Surg Pathol. 2002;26(9):1095-1110.

7. Van Osdol AD, Landercasper J, Andersen JJ, et al. Determining whether excision of all fibroepithelial lesions of the breast is needed to exclude phyllodes tumor: upgrade rate of fibroepithelial lesions of the breast to phyllodes tumor. JAMA Surg. 2014;149(10):1081-1085.

8. Piscuoglio S, Geyer FC, Burke KA, et al. Massively parallel sequencing analysis of synchronous fibroepithelial lesions supports the concept of progression from fibroadenoma to phyllodes tumor. NPJ Breast Cancer. 2016;2:16035.

9. Lim WK, Ong CK, Tan J, et al. Exome sequencing identifies highly recurrent MED12 somatic mutations in breast fibroadenoma. Nat Genet. 2014;46(8):877-880. 
10. Piscuoglio S, Murray M, Fusco N, et al. MED12 somatic mutations in fibroadenomas and phyllodes tumours of the breast. Histopathology. 2015;67(5):719-729.

11. Cani AK, Hovelson DH, McDaniel AS, et al. Next-Gen Sequencing Exposes Frequent MED12 Mutations and Actionable Therapeutic Targets in Phyllodes Tumors. Mol Cancer Res. 2015;13(4):613-619.

12. Piscuoglio $\mathrm{S}, \mathrm{Ng} \mathrm{CK}$, Murray $\mathrm{M}$, et al. Massively parallel sequencing of phyllodes tumours of the breast reveals actionable mutations, and TERT promoter hotspot mutations and TERT gene amplification as likely drivers of progression. $J$ Pathol. 2016;238(4):508-518.

13. Noguchi S, Yokouchi H, Aihara T, et al. Progression of fibroadenoma to phyllodes tumor demonstrated by clonal analysis. Cancer. 1995;76(10):1779-1785.

14. Kuijper A, Buerger $\mathrm{H}$, Simon $\mathrm{R}$, et al. Analysis of the progression of fibroepithelial tumours of the breast by PCR-based clonality assay. J Pathol. 2002;197(5):575-581.

15. Ng CKY, Di Costanzo GG, Tosti N, et al. Genetic profiling using plasma-derived cellfree DNA in therapy-naive hepatocellular carcinoma patients: a pilot study. Ann Oncol. 2018;29(5):1286-1291.

16. Landercasper $\mathrm{J}$, Linebarger $\mathrm{JH}$. Contemporary breast imaging and concordance assessment: a surgical perspective. Surg Clin North Am. 2011;91(1):33-58.

17. Schoonjans JM, Brem RF. Fourteen-gauge ultrasonographically guided large-core needle biopsy of breast masses. J Ultrasound Med. 2001;20(9):967-972.

18. Gould DJ, Salmans JA, Lassinger BK, et al. Factors associated with phyllodes tumor of the breast after core needle biopsy identifies fibroepithelial neoplasm. J Surg Res. 2012;178(1):299-303.

19. Tan PH TG, Lee A, et al. . Fibroepithelial tumours In: Lakhani SR El, Schnitt SJ, et al, ed. WHO Classification of Tumours of the Breast, 4th edn. 4th ed. : IARC Press: Lyon, France, 2012. . 
20. Yoshida M, Ogawa R, Yoshida $\mathrm{H}$, et al. TERT promoter mutations are frequent and show association with MED12 mutations in phyllodes tumors of the breast. Br J Cancer. 2015;113(8):1244-1248.

21. Pareja F, Geyer FC, Kumar R, et al. Phyllodes tumors with and without fibroadenomalike areas display distinct genomic features and may evolve through distinct pathways. NPJ Breast Cancer. 2017;3:40.

22. Abe M, Miyata S, Nishimura S, et al. Malignant transformation of breast fibroadenoma to malignant phyllodes tumor: long-term outcome of 36 malignant phyllodes tumors. Breast Cancer. 2011;18(4):268-272.

23. Sanders LM, Daigle ME, Tortora M, Panasiti R. Transformation of benign fibroadenoma to malignant phyllodes tumor. Acta Radiol Open. 2015;4(7):2058460115592061.

24. Telli ML, Horst KC, Guardino AE, Dirbas FM, Carlson RW. Phyllodes tumors of the breast: natural history, diagnosis, and treatment. J Natl Compr Canc Netw. 2007;5(3):324-330. 


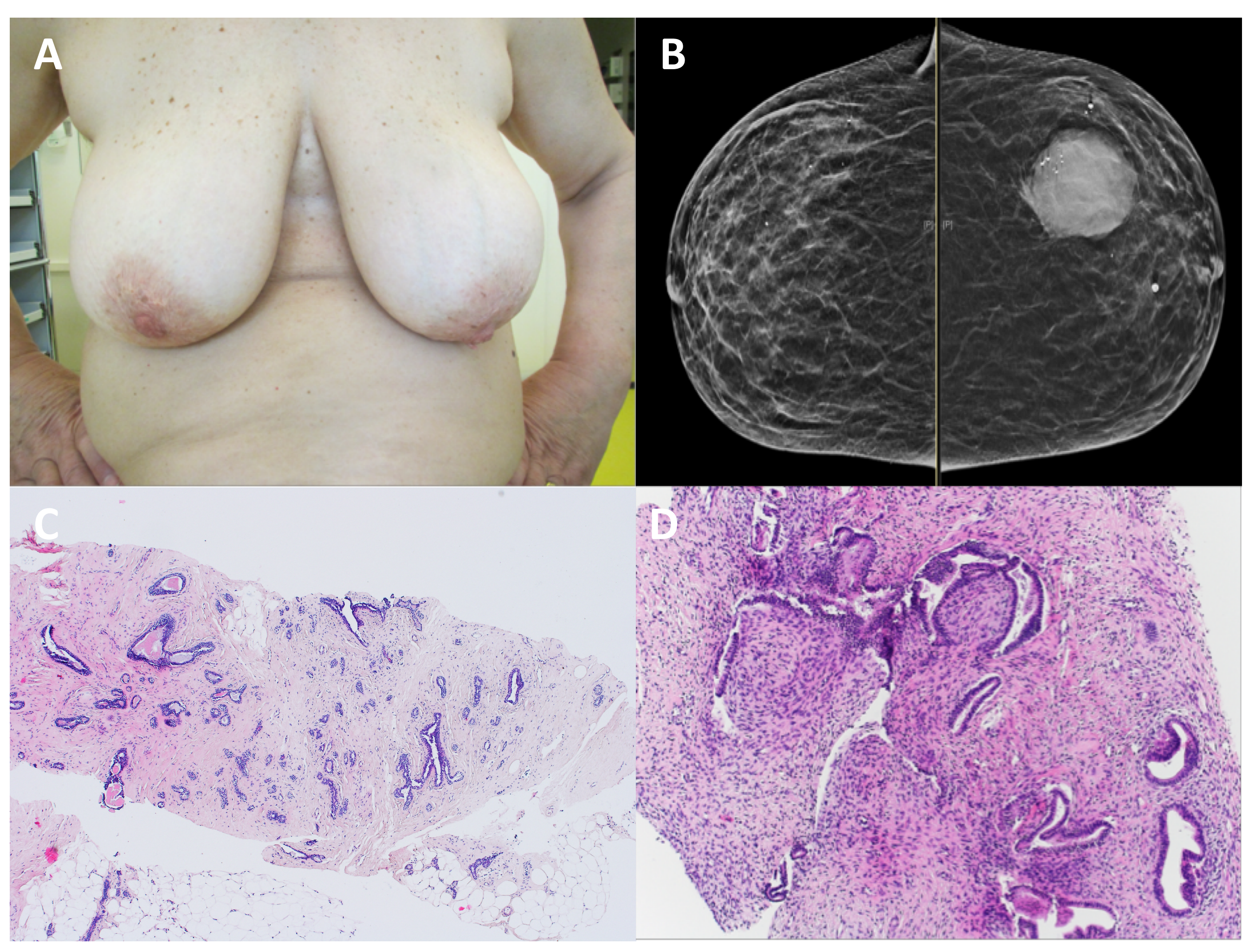




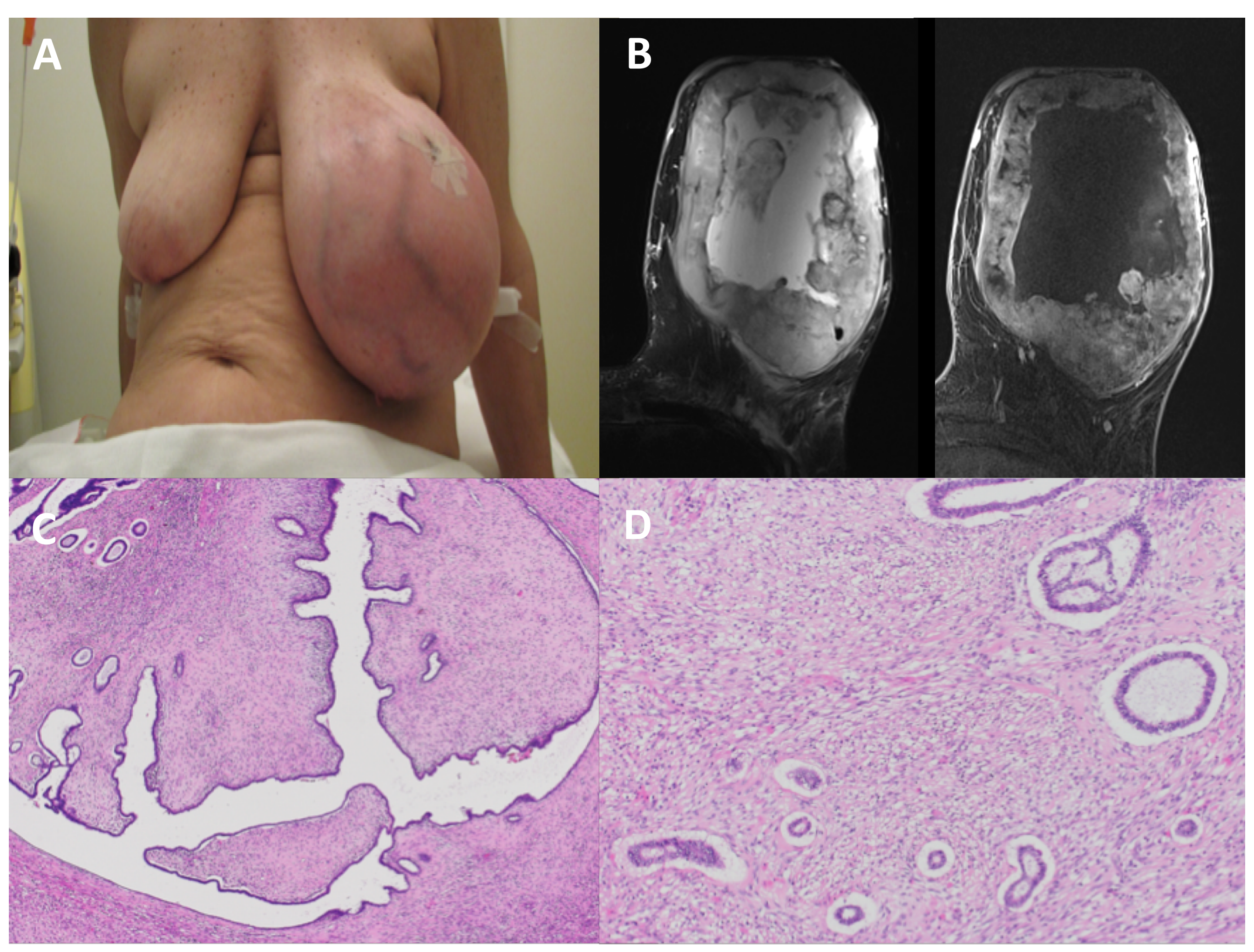




\section{Figure 3}

A

\section{B2 lesion}
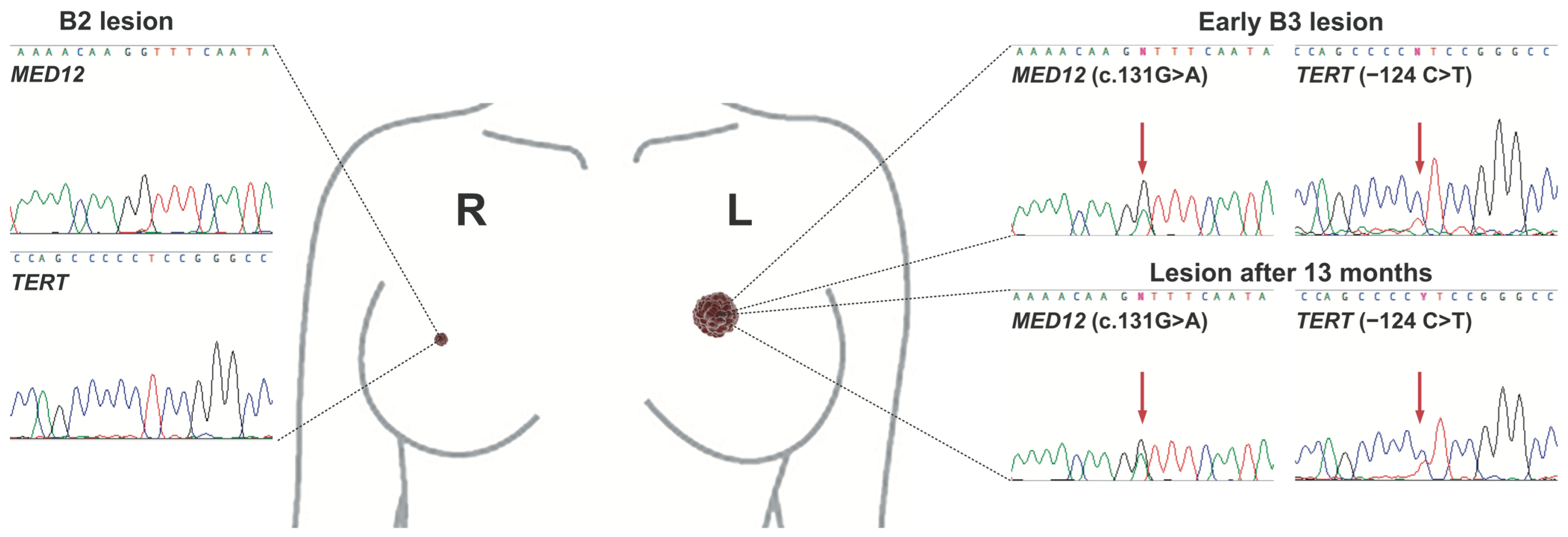

B

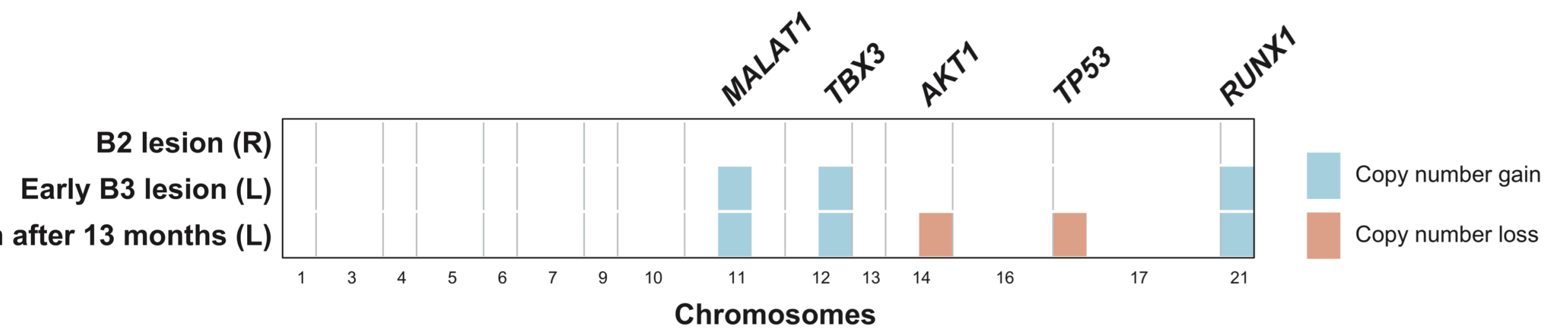




\section{Figures Legend}

Figure 1: Clinical, radiological and histological images at first presentation

A) Photo of the breast at first presentation

B) Mammogram showing a $55 \times 50 \mathrm{~mm}$ mass on the left breast and a $6 \times 7 \mathrm{~mm}$ mass on the right breast

C) Core needle biopsy of the right breast lesion showing a fibroepithelial lesion well demarcated from the surrounding tissue with low stromal cellularity, consistent with fibroadenoma (magnification 40x, H\&E)

D) Core needle biopsy of the left breast lesion showing a fibroepithelial lesion with mildly increased stromal cellularity but no stromal cell atypia (magnification 100x, H\&E).

Figure 2: Clinical, radiological and histological images 13 months after the first presentation

A) Photo of the breast after 13 months

B) Left: Axial fluid sensitive T2 - weighted MR image (TIRM). Right: Axial contrast-enhanced T1-weighted fat-suppressed MR image. Both images showing a $16 \mathrm{~cm}$ heterogeneous mass filling the entire left breast with an extensive central fluid collection

C) Surgical specimen (mastectomy) of the left breast: showing a borderline phyllodes tumor with typical intracanalicular "leaf-like" growth pattern and mildly increased, variable stromal cellularity (magnification 40x, H\&E)

D) Surgical specimen (mastectomy) of the left breast: areas reminiscent of a cellular fibroadenoma without stromal cellatypia (magnification 100x, H\&E).

\section{Figure 3: Genetic profiling of the lesions}

A) Sanger sequencing for the MED12 hotspot and for TERT promoter mutations. No somatic mutation was identified in the FA (right breast) whereas MED12 hotspot (c.131G >A) and TERT promoter (c.-124C>T) mutations were identified in both the early B3 lesion and in the PT (left breast).

B) Copy number profiling of the 29 complete coding genes included in the targeted sequencing panel. No copy number alteration was identified in the FA (right breast). Gains of MALAT1, TBX3 and RUNX1 were found in both the early B3 lesion and in the PT (left breast). Addition losses of AKT1 and TP53 were found only in the lesion after 13 months. 